\title{
Neural Model of Cortical Dynamics in Resonant Boundary Detection and Grouping
}

\author{
Heiko Neumann, Petra Mössner
}

\author{
Abt. Neuroinformatik, Fakultät $f$. Informatik, Universität Ulm, \\ D-89069 Ulm, Germany; hneumann@neuro.informatik.uni-ulm.de
}

\begin{abstract}
A new model for visual boundary detection and contour grouping is presented that is based on functional elements of resonant matching of activation between neural layers in cortical architecture. The model architecture relates to visual cortical areas V1 and V2 which are bidirectionally interconnected via feedforward as well as feedback projections. It is suggested that their functionality is primarily determined by the measurement and integration of signal features that are continuously matched against neural codes of expectancies generated by the long-range integration of coherent activity. The net effect produces grouping and illusory contour completion at model V2 as well as contextsensitive shaping of orientation tuning and selectivity of receptive fields at model V1 layer. A pilot implementation of the model architecture has been successfully tested on various test stimuli.
\end{abstract}

\section{Introduction}

Grouping of visual elements of fragmented layout is a key functional mechanism for the segmentation of surface outlines. The detection of boundary contours contributes to the generation of an abstract representation of the visual scene. Several approaches to grouping have been developed to model neural mechanisms in biological and computer vision (e.g. $[6,4,7,12]$ and $[11,14]$ ).

We propose a novel functional architecture that has been inspired by the principles of adaptive resonance theory ([5]). The architecture consists of two major stages of feedforward and feedback processing each of which having different sublayers. The input to the recurrent network of resonant processing is generated by orientation selective units for local contrast detection. Subsequent processing is organized as a bidirectionally connected structure of $F_{1}$ and $F_{2}$ layers, which functionally correspond to cortical levels V1 and V2, respectively. Afferent activity generated at V1 feeds forward to V2 where it is integrated from different spatial branches in the visual field. Conjunctive arrangement of oriented input activity in turn generates an activation that is fed back to gate activities of orientation selective units in V1. This mechanism implies a new computational theory in which V2 acts as a code layer such that the input signal activation in V1 is tested against "expected" activity in a much broader visual context $([5,10])$. The gating mechanism is closely related to the hypothesized linking fibres suggested to model mechanisms of activity synchronization for feature linking (e.g. [1]). The net effect of gating and positional and orientational competition shapes the orientation selectivity of cells in a context-sensitive 
manner ([3]). Furthermore, inconsistent activity that fails to match the V2 feedback "expectancy" will be suppressed. Contributions to grouping and long-range completion are thus predicted to be generated at the level of V2 ([13]) primarily due to increased receptive field size and non-linear input integration.

\section{Outline of the Computational Architecture}

The input activity distribution to the considered functional architecture is generated by oriented contrast detection at the complex cell level (denoted by $x_{i \varepsilon}$, for location $i$ and orientation $\varepsilon$ ). The specific mechanisms for their generation will not be discussed here (but see e.g. [9]). The architecture consists of two main network layers, namely $F_{1}$ and $F_{2}$ (see Fig. 1(a)), each of which is organized in two sublayers. Processing in $F_{1}$-layer currently consists of two processing stages: (1) Local competition (on-center/off-surround feedback processing) generates activity based on resonant matching between feedforward and feedback streams, and (2) local competition of "match activity" from the previous stage to enhance spatial and orientation contrast. In the first sublayer $w_{i \varepsilon}^{(1)}$-activity denotes the activation generated in response to the combination of the bottom-up feedforward (denoted by $x$-activities) and the top-down feedback stream (denoted by $y$-activities):

$$
\frac{d}{d t} w_{i \varepsilon}^{(1)}=-D w_{i \varepsilon}^{(1)}+\left(U-w_{i \varepsilon}^{(1)}\right)\left(x_{i \varepsilon}+C x_{i \varepsilon} f\left(y_{i \varepsilon}^{(2)}\right)\right)-w_{i \varepsilon}^{(1)} \sum_{j \in \mathcal{N}_{i}^{y}}\left(\sum_{\gamma} f\left(y_{j \gamma}^{(2)}\right) \cdot \psi_{\bar{\varepsilon} \gamma}^{-}\right) \cdot \lambda_{i j}^{(y-)},
$$

where $f($.$) denotes a signal function for the transformation of feedback activity.$ In the next sublayer $w_{i \varepsilon}^{(2)}$-activity is defined by

$$
\frac{d l}{d t} w_{i \varepsilon}^{(2)}=-E w_{i \varepsilon}^{(2)}+\left(F-G w_{i \varepsilon}^{(2)}\right) \sum_{\gamma} w_{i \gamma}^{(1)} \cdot \psi_{\varepsilon \gamma}^{+}-w_{i \varepsilon}^{(2)} \sum_{j \in \mathcal{N}_{i}^{w}}\left(\sum_{\gamma} w_{j \gamma}^{(1)} \cdot \psi_{\varepsilon \gamma}^{-}\right) \cdot \lambda_{i j}^{(w-)}
$$

The constants $D$ and $U$ in eqn. 1 and $E, F$ and $G$ in eqn. 2 denote the parameters of the shunting equations to control passive decay and saturation levels. Equation 2 is solved at equilibrium for $G=0$. One-dimensional weighting functions $\psi_{\varepsilon \gamma}^{ \pm}$, both centered at target orientations (with $\psi_{\varepsilon \gamma}^{+}$more narrowly tuned than $\psi_{\bar{\varepsilon} \gamma}^{-}$) realize an on-center/off-surround interaction scheme in orientation space between orientations $\varepsilon$ and $\gamma$. The inhibitory weighting function $\lambda_{i j}^{(w-)}$ realizes a spatial coupling (low-pass filter) for locations $j$ in the neighborhood of $i$. The constant $C$ (eqn. 1) denotes a scaling factor for the transformed excitatory feedback activation.

In the subsequent $F_{2}$-layer curvilinear arrangements of oriented contrast activation will be integrated based on the evaluation of a much broader spatial context. Processing in $F_{2}$-layer again consists of two hierarchically organized sublayers: (1) Long-range cooperative interaction integrates contrast activation in a curvilinear arrangement, and (2) local intra-orientational on-center/off-surround competition (between spatial locations) and activity normalization over orientations generate a locally contrast-enhanced activation distribution. The integration mechanism at the first $F_{2}$-sublayer realizes a spatial relatability measurement 


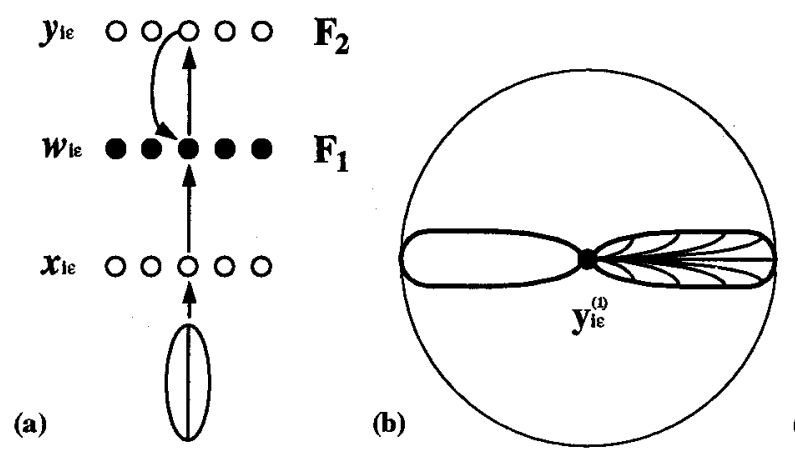

(c)

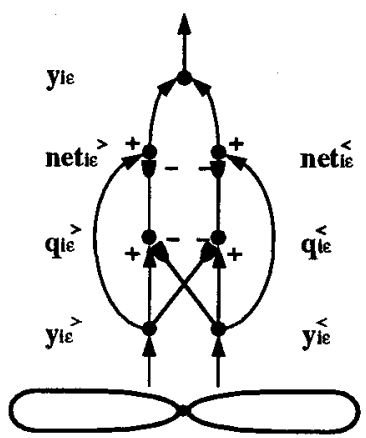

Fig. 1. Components of the neural architecture; (a) layout of coarsely defined model structure with basic network layers $F_{1}$ and $F_{2}$, (b) definition of spatial layout of bipole cells (the target cell receives input from two spatial subfields). (c) definition of the micro-circuit which implements the spatial interaction scheme for activity accumulation from different branches.

(see [8]). The mechanism is based on a bipole cell (Fig. 1(b), see e.g. $[6,7]$ ) in which two non-linearly interacting subfields integrate activity from two different half-planes in the visual field. The integration utilizes an 'association field' ([2]) which is subdivided into two spatially coincident antagonistic ON- and OFFsubfields. The support of a given activation in an orientation field is determined on the basis of an evaluation of a compatibility function such as in probabilistic relaxation schemes ([15]). The spatial outlines of the bipole integration fields are displayed in Fig.2. The activities $y_{i \varepsilon}$ and $y_{i \varepsilon}^{\leftarrow}$ represent the spatially integrated orientation responses in the left and right subfield of the bipole, respectively. The cooperation mechanism depicted in Fig. 1(c) is realized by a micro-circuit of non-linear feedforward disinhibitory interaction of conjunctive input activation. Pooling of equilibrated responses of individual bipole branches at the final stage is denoted by an additive equation, namely $\frac{d}{d t} y_{i \varepsilon}^{(1)}=-y_{i \varepsilon}^{(1)}+\operatorname{net}_{i \varepsilon} \overrightarrow{+n e t} \overleftarrow{i \varepsilon}$. At steady-state, the net response for the non-linear integration results in

$$
y_{i \varepsilon}^{(1)}=y_{i \varepsilon} \overrightarrow{i \varepsilon} \frac{\frac{2}{B}+y_{i \varepsilon}+y_{i \varepsilon}^{\overleftarrow{i \varepsilon}}}{\frac{2}{B^{2}}+\frac{1}{B}\left(y_{i \varepsilon}+y_{i \varepsilon}\right)+y_{i \varepsilon} y_{i \varepsilon}} .
$$

The constant $B$ is used to control the effectivity of interactions between both input branches. This demonstrates that the bipole generates a net response only if both subfields receive positive net input activation.

The processing stage in the second sublayer consists of a spatial competition between activations in a local spatial neighborhood over all orientations. Thus the individual activity generated at one position for one orientation is normalized w.r.t. the spatially blurred activation of orientation columns. The resulting activity is defined by

$$
\frac{d}{d t} y_{i \varepsilon}^{(2)}=-H y_{i \varepsilon}^{(2)}+\left(K-M y_{i \varepsilon}^{(2)}\right) y_{i \varepsilon}^{(1)}-y_{i \varepsilon}^{(2)} \sum_{j \in \mathcal{N}_{i}^{y}} \sum_{\gamma} y_{j \gamma}^{(1)} \cdot \lambda_{i j}^{(y-)} .
$$


The constants $H, K$ and $M$ denote the parameters of the shunting equation. Equation 4 is solved at equilibrium with $M=0$. This activity generated in the $F_{2}$-layer is fed back to layer $F_{1}$ to match oriented contrast measures $x_{i \varepsilon}$ and generate $w_{i \varepsilon}^{(1)}$-activations.
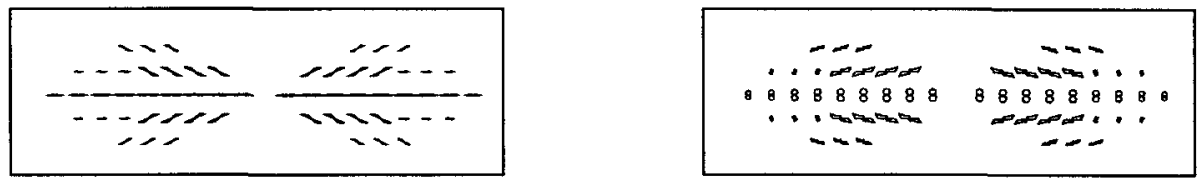

Fig. 2. Outline of spatial integration in bipole cells based on a spatial weighting function ('association field') and a compatibility function in the feature domain; ON-field (left) denotes spatial orientations that support the target orientation (bipole center) in an excitatory fashion, OFF-field (right) denotes orientations which activations provide inhibitory contributions to the support function.

\section{Simulation Results}

The new proposed network architecture has been implemented and tested on a set of stimuli. All linear equations have been solved at equilibrium; Eqn.1 has been solved numerically using a 4 th order Runge Kutta scheme with adaptive step size control.

In this initial phase of the model evaluation, input data has been synthesized at the complex cell stage ( $x$-activities). In particular, the input energy at each spatial location is equally distributed over all orientations of the orientation field. Hence, such an initialization generates maximal orientation uncertainty in a model hypercolumn at a given spatial location. We tested the competence of the network for different spatial arrangements of input activation. The goal of this initial testing was to evaluate the self-organizing properties of the network response based on the spatial arrangement of salient configurations of input activation alone. Figure 3 shows the results of processing for a rectangular pattern of input activity (left). The equilibrated net activations in the $F_{1}$ and $F_{2}$ layers are shown. The final representation shows that properties such as activity suppression in homogeneous regions, border enhancement and context sensitive shaping of receptive field sensitivity occur at the model V1 stage $\left(F_{1}\right)$. At the model V2 stage $\left(F_{2}\right)$ cells are selective to orientations of the shape outline. A line grating pattern is used as input in Fig.4. The results show the influence of context sensitivity on RF profiles and the ability to bridge boundary gaps. Thus, the network is capable to generate activity patterns that correspond to illusory contours.

\section{Summary}

A new model architecture has been developed for visual boundary detection and grouping. The functional organization is based on the principles of adaptive re- 
sonance. We postulate that the role of V2 $\rightarrow \mathrm{V} 1$ feedback processing can be understood as a process of dynamic code testing and prediction. The net effect produces grouping and illusory contour completion. In particular, the results of model simulations demonstrate that (1) the orientation selectivity of V1 cells is sharpened depending on the spatial arrangement and visual context, (2) orientation selective contrast measurements are enhanced at boundaries of spatially homogeneous stimulus arrangements and suppressed in the interiors, (3) oriented cells at model V2 stage only respond to curvilinear input arrangements of activity at both branches of bipole cells, (4) model V2 cells show fine tuning for orientation selectivity and generate subjective contours to bridge gaps in arrangements of oriented contrast. Based on these initial test cases we intend to systematically investigate preprocessed input luminance patterns of the type used as stimuli in psychophysical experiments.

\section{References}

1. R. Eckhorn, H.J. Reitboeck, M. Arndt, and P. Dicke. Feature linking via synchronization among distributed assemblies: Simulations of results from cat visual cortex. Neural Computation, 2:293 - 307, 1990.

2. D.J. Field, A. Hayes, and R.F. Hess. Contour integration by the human visual system: Evidence for local 'association field'. Vision Research, 33(2):173 - 193, 1993.

3. C.D. Gilbert and T.N. Wiesel. The influence of contextual stimuli on the orientation selectivity of cells in primary visual cortex of the cat. Vision Rescarch, 30(11):1689 - 1701, 1990.

4. A. Gove, S. Grossberg, and E. Mingolla. Brightness perception, illusory contours and corticogeniculate feedback. Visual Neuroscience, 1995. (in press).

5. S. Grossberg. How does a brain build a cognitive code? Psychological Review, 87:1 $-51,1980$.

6. S. Grossberg and E. Mingolla. Neural dynamics of perceptual grouping: Textures, boundaries, and emergent segmentation. Perception and Psychophysics, 38(2):141 $-171,1985$.

7. F. Heitger and R. von der Heydt. A computational model of neural contour processing: Figure-ground segregation and illusory contours. In Proc. 4th Int. Conf. on Computer Vision, ICCV-93, Berlin, May 11-14 1993.

8. P.J. Kellman and T.F. Shipley. A theory of visual interpolation in object perception. Cognitive Psychology, 23(2):141 - 221, 1991.

9. J.J. Koenderink and A.J. van Doorn. Generic neighborhood operators. IEEE Transactions on Pattern Analysis and Machine Intelligence, PAMI-14(6):597 - 605, 1992.

10. D. Mumford. On the computational architecture of the neocortex II: The role of cortico-cortical loops. Biological Cybernetics, 65:241 - 251, 1991.

11. P. Parent and S.W. Zucker. Trace inference, curvature consistency, and curve detection. IEEE Transactions on Pattern Analysis and Machine Intelligence, PAMI11(8):823 - 839, 1989.

12. W.D. Ross, S. Grossberg, and E. Mingolla. A neural model of illusory contour formation in V1 and V2. In Proc. ARVO'95 (Investigative Ophtalmology and Visual Science, PGM\# 2187), 1995.

13. R. von der Heydt and E. Peterhans. Mechanisms of contour perception in monkey visual cortex. I. Lines of pattern discontinuity. The Journal of Neuroscience, $9(5): 1731-1748,1989$. 
14. S.W. Zucker, A. Dobbins, and L. Iverson. On the computational neurobiology of curve detection. In Proc. British Machine Vision Conference (BMVC90), pages xvii - xxiii, Oxford (GB), Sept. 24-27 1990.

15. S.W. Zucker, R.A. Hummel, and A. Rosenfeld. An application of relaxation labeling to line and curve enhancement. IEEE Transactions on Computers, C-26(4):394 $-403,1977$.
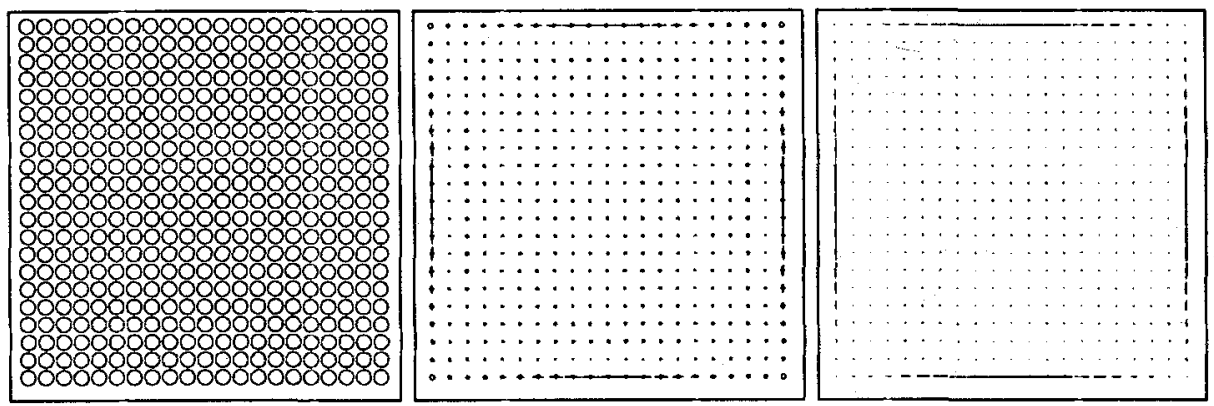

Fig. 3. Processing results for a rectangular shape: Spatial input activation of equal magnitude for all orientations of a hypercolumn (left); equilibrated $w^{(2)}$-activity in $F_{1}$ layer displays context dependent tuning of cells along borders of the square arrangement, orientation uncertainties at corners and reduced activity in the interior (center); equilibrated $y^{(2)}$-activity in $F_{2}$ layer displays computed grouping information (after bipole integration) fed back to $F_{1}$ layer (right). Note, that the reduction of activity near corners depends solely on the shape of the spatial coupling in the bipole for activity integration.
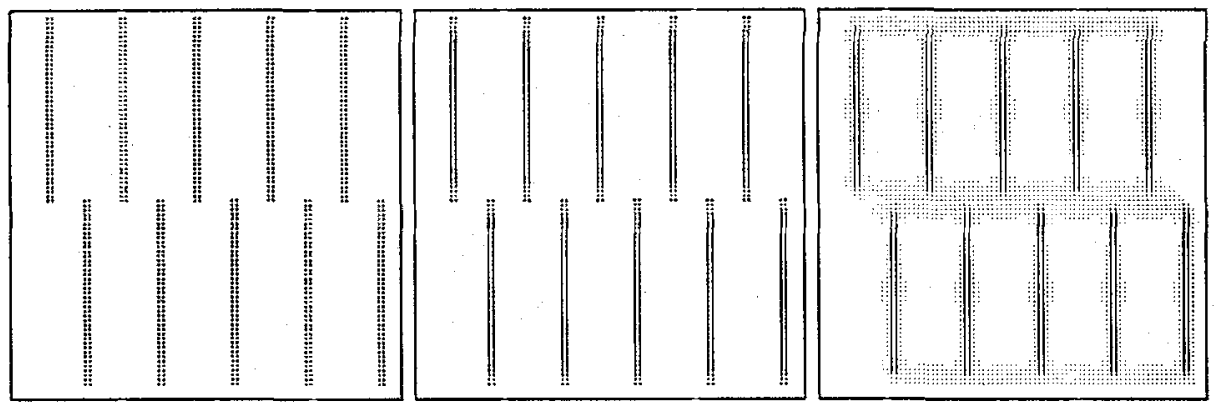

Fig. 4. Processing results for a bar grating: Spatial arrangement of a synthetically generated distribution of input activity (left); $w^{(2)}$-activity distribution in $F_{1}$ layer (center) shows minimized orientation uncertainty for individual bars with uncertainty at line ends; $y^{(2)}$-activity distribution in $F_{2}$ layer (right) displays strong grouping activity along bar outlines and more shallow integration along illusory contour completion orthogonal to line ends. 